\title{
FUNDAMENTOS TEÓRICOS DE LA ESTRATEGIA ORGANIZACIONAL COMO TÉCNICA PARA FORTALECER EL PLAN DE CAPACITACIÓN DEL TALENTO HUMANO EN LA EMPRESA MODERNA
}

\section{Theoretical Foundations of the Organizational Strategy as a Technician of the Human Talent Training Plan in Modern Business}

\author{
Zea Vallejo Daniel Arturo, Ing. \\ Universidad de Guayaquil, Ecuador \\ https://orcid.org/0000-0001-6360-7991 \\ daniel.zeav@ug.edu.ec
}

\author{
Alonzo Manzano Katherine Estefania, Ing. \\ Universidad de Guayaquil, Ecuador \\ https://orcid.org/0000-0002-9947-1966 \\ katherine.alonzom@ug.edu.ec
}

Palabras claves: Estrategias Organizaciones, Técnica, Plan de Capacitación para el Personal. Recibido: 28 de septiembre de 2020

Keywords: Organizational Strategies, Technique, Staff Training Plan.

Aceptado: 11 de diciembre de 2020

\section{RESUMEN}

El presente estudio se planteó el objetivo de describir los Fundamentos Teóricos de la Estrategia Organizacional como Técnica para fortalecer el Plan de Capacitación del Talento Humano en la Empresa Moderna. La investigación se realizó bajo la perspectiva documental-descriptiva. Como resultado se obtuvo que la adopción de estrategias organizacionales para la capacitación del talento humano debe comprender propuestas viables en su implementación las cuales deben dirigirse, entre otros aspectos, a fortalecer el plan de capacitación para las diversas áreas de la empresa, atendiendo a temas relevantes como lo son: la tecnología, crecimiento personal, motivación y aprendizaje. En conclusión, las estrategias organizacionales están conformadas por cuatro fases claves objeto de ser incorporadas, ellas son: un diagnóstico, diseño, implementación y evaluación. Las estrategias organizacionales no deben ser entendidas como un cuerpo rígido de pasos inviolables sin otras alternativas de realización creativa.

\begin{abstract}
The present study set out the objective of describing the Theoretical Foundations of the Organizational Strategy as a Technique to strengthen the Human Talent Training Plan in Modern Enterprise. The research was carried out under the documentary-descriptive perspective. As a result, it was obtained that the adoption of organizational strategies for the training of human talent should include viable proposals in its implementation which should be directed, among other aspects, to strengthen the training plan for the various areas of the company, taking into account relevant issues as they are: technology, personal growth, motivation and learning. In conclusion, the organizational strategies are made up of four key phases that are to be incorporated, they are: a diagnosis, design, implementation and evaluation. Organizational strategies should not be understood as a rigid body of inviolable steps without other creative realization alternatives.
\end{abstract}




\section{INTRODUCCIÓN}

Durante años, capacitación y adiestramiento tuvieron connotaciones asociadas al aprendizaje mecánico. En este sentido, Frody (2016), explica que "Hoy en día, es claro que se trata de un enfoque sistemáticamente planeado para adquirir conocimientos, capacidades y actitudes, a partir de ciertos elementos relevantes" (p. 45). Además, es un proceso tendiente a cambiar conductas y motivaciones para armonizar las características de una persona con las demandas de un puesto (ob cit).

Según lo anterior, ese proceso consiste en planes conformados por estrategias organizacionales agrupadas en técnicas diseñados para mejorar competencia y desempeño en escala individual, grupal y organizacional (Frody, 2016). A su vez, ello implica cambios mensurables en aspectos como: conocimientos, aptitudes, capacidades, conductas y motivaciones. El móvil primario de la enseñanza en una organización es modificar el desempeño del personal en todos los niveles. (ob cit)

En tal ámbito de ideas, tal y como afirma Carruzo (2015), "se busca más ganancias y los accionistas más dividendos aumentando ventas, reduciendo costos operativos o haciendo ambas cosas al mismo tiempo" (p. 30). Al respecto, Directivos y ejecutivos afrontan continuas presiones para dar servicios más eficaces y eficientes. Por ello, las empresas deben responder a esos retos, advirtiendo que los avances en ciencia y tecnología desactualizan muchas aptitudes tradicionales. Simultáneamente, ello entraña desarrollar nuevas capacidades (ob cit).

Por lo tanto, el éxito final de cualquier organización depende de que su talento humano lleve a cabo bien las tareas actuales y, entretanto, sepan adaptarse a nuevas situaciones (Carruzo, 2015). En este sentido, el aprendizaje puede ser clave para mejorar el desempeño de un empleado ya desde sus primeros días en la organización (ob cit).

Considerando lo expuesto, el estudio desarrollado se caracteriza, en primer lugar, por su plena correspondencia con las sugerencias expuestas por especialistas que señalan la importancia del uso de las estrategias organizacionales en los planes y programas como una técnicas que puede fortalecer procesos imprescindibles en las organizaciones modernas, la capacitación de su personal es uno de ellos (Carruzo, 2015), por tanto su novedad no radica en que modifica lo orientado al respecto, sino en que ofrece una vía para materializar tales orientaciones (ob cit). La misma no debe ser entendida como un cuerpo rígido de pasos inviolables sin otras alternativas de realización creativa. Por lo que todo lo que se propone a continuación, debe ser asumido como una guía para la acción, que no cierre las puestas a la concepción y realización de otras actividades (Frody, 2016).

En este sentido, la estrategia organizacional se centra en dos conceptos; el primero, la consolidación de los objetivos estratégicos inherentes a toda organización: supervivencia, crecimiento y rentabilidad; el segundo, la creación de valor, innovación y desarrollo tecnológico (Frody, 2016). Desde una perspectiva etimológica puede afirmarse que el concepto estrategia tiene dos momentos determinantes y bien diferenciados: el primero, relativo a su génesis en torno a la cual existe unificado criterio por parte de los miembros de la comunidad académica respecto al reconocimiento de ella en el escenario militar (ob cit). El segundo, el que corresponde a su uso deliberado en el contexto organizacional relacionado con la concepción moderna de él, cuyo fundamento es la toma decisiones sobre objetivos, metas y recursos, a partir de la estimación de las condiciones presentes y futuras, endógenas y exógenas, favorables y desfavorables a la organización (Frody, 2016).

Por su parte, Barreto (2010), manifiesta que:

... las estrategias organizacionales en la capacitación del talento humano en la empresa moderna consideran imprescindible crear todas las condiciones que permitan mejorar las competencias laborales en los empleados de la organización, en este sentido, está íntimamente relacionada con la manera en que se concibe al sujeto de la capacitación, el cual posee una experiencia y un valioso conocimiento por lo que a la vez que es receptor, es también emisor de conocimiento (p. 134).

Según lo anterior, resaltan el proceso de aprendizaje activo y dinámico, considera a la capacitación en función de enseñar a reflexionar de forma creadora e innovadora al sujeto a través de su práctica para lograr una nueva práctica transformadora (Barreto, 2019). A partir de estos fundamentos se repotencia el mejoramiento de las competencias, de manera que se eleva la efectividad de su desempeño y con ello mejora el nivel de atención a la clientela (ob cit).

En este sentido, las acciones en la estrategia deben desarrollarse sobre la base de métodos de trabajo que propicien la búsqueda de desarrollo próximo para hacer real el desarrollo potencial de los trabajadores (Frody (2016), donde los procesos de acompañamiento sistemático a la acción del trabajador se convierten en mediador simbólico para alcanzar el estado deseado (Carruzo, 2015). 
Es por ello que el objetivo fundamental de esta investigación es analizar los fundamentos teóricos de la estrategia organizacional como técnica para fortalecer el plan de capacitación del talento humano en la empresa moderna. Para cumplir con los objetivos trazados se utilizó el método investigativo cualitativo y como técnicas para la recolección de la información fueron el análisis y síntesis, fichaje, revisión de literatura especializada.

\section{DESARROLLO}

\section{Organización}

En relación a la organización, puede destacarse que es la función que consiste en buscar los medios que son necesarios poner en práctica para determinar el grado de eficiencia del órgano administrativo, su rentabilidad, así como su facultad de adaptarse al medio ambiente (Aquino, (2016). Al respecto, la organización administrativa como lo expone Carruzo (2015), "es la que agrupa las actividades necesarias para realizar lo planeado" (p. 17).

En este sentido, las organizaciones son muy variables, según el grado de su estructuración puede ser formal e informal (Aquino, 2016). La organización formal, es la que aparece trazada en los diagramas de organización. Barreto (2010), manifiesta que "Es la estructura formalmente establecida por los administradores, quienes definen las tareas que deben ser ejecutadas y asignan responsabilidades para su ejecución" (p. 45). La informal es aquella que emerge espontánea entre las personas que ocupan posiciones en la organización formal, a partir de las relaciones humanas establecidas al desempeñarse en sus cargos (ob cit).

\section{Talento Humano}

El talento humano tiene una importancia considerable dentro de las organizaciones, al respecto, Aquino (2016), opina que:

... brindan creatividad y chispa, se encargan de producir bienes y servicios, controlan los aspectos de la calidad, más aún orientan estos productos hacía un mercado y establecen de la manera más expedita las estrategias para que este producto sea aceptado por los consumidores, sin duda alguna las personas, es decir el talento, es quien realmente le da valor a las organizaciones (p. 127)

En este margen de ideas, la gestión del talento humano es un área muy sensible a la mentalidad que predomina en las organizaciones (ob cit). Un aspecto significativo a tomar en cuenta es que es contingente y situacional, por cuanto depende de aspectos tales como la cultura de cada organización, la estructura organizacional adoptada, de la misma manera los caracteres del contexto ambiental, el negocio de la organización, la tecnología utilizada, los procesos internos y otra infinidad de variables importantes. (Aquino, 2016)

\section{Capacitación del Talento Humano en la Empresa Moderna}

La capacitación del talento humano en la empresa moderna es un proceso continuo y permanente, Dolan, Schuler, y Valle, (2011), escriben que obedece "a necesidades primeramente individuales en función al perfil del profesional y las necesidades del puesto de trabajo y el equipo a donde pertenece" (p. 87). En este sentido, este proceso cosiste en la información, conocimiento y desarrollo de destrezas que se brinda a un individuo, para que este pueda complementar su propio conocimiento y desarrollarlo en el tiempo, (Chiavenato, 2013), siendo capaz de proponer métodos de trabajo, iniciativa y mejorar sustancialmente su desempeño en el ambiente laboral (ob cit). Puede definirse de la siguiente manera, según los autores citados:

Un proceso educativo de corto plazo, aplicado de manera sistemática y organizada, por medio del cual las personas adquieren conocimientos, desarrollan habilidades y competencias en función de los objetivos previamente definidos. La capacitación entraña la trasmisión de conocimientos específicos relativos al puesto de trabajo, actitudes frente aspectos de la organización, de la tarea y del ambiente, así como el desarrollo de competencias. (p. 217)

Según lo anteriormente expuesto, este proceso representa entonces las actividades que desde el seno de una organización, se adecuan en función de corresponder a las necesidades particulares de los empleados, buscando mejorar la calidad en su desempeño, en la actitud, sus habilidades y conductas personales, para maximizar su eficacia en el logro de las metas que se ha propuesto la organización (Dolan, Sehuler, y Valle, 2011).

Por su parte, la capacitación del talento humano propone la enseñanza de conocimientos claves, específicos, nuevos, suministrando herramientas fundamentales que permiten empoderar al participante, actualizando muchas veces conocimientos ya adquiridos, pero que han sufrido modificaciones en cuanto a las técnicas y métodos para ejecutarlos (Dolan, Sehuler, y Valle, 2011). 


\section{Importancia de la Capacitación del Talento Humano en la Empresa Moderna}

Un aspecto significativo a citar es que la capacitación del talento humano en las organizaciones es de vital importancia porque contribuye al desarrollo de los colaboradores tanto personal como profesional (Barthes, 2009). Por ello las empresas deben encontrar mecanismos que den a sus colaboradores los conocimientos, habilidades y actitudes que se requiere para lograr un desempeño óptimo (ob cit). Siden (2014), sostiene que:

Así como en algún momento la tierra dejó de ser la principal fuente de riquezas para pasar a serlo las industrias con sus máquinas, hoy las máquinas van dejando el primer lugar a los productores de conocimientos. En este contexto, en este nuevo paradigma productivo, la capacitación y el desarrollo son áreas claves y de vital importancia en las organizaciones (p. 93).

Es decir, que según lo reseñado en la cita, el tema de la capacitación del talento humano para las empresas en la actualidad es importante (ob cit), ya que contribuye al desarrollo personal y profesional de los individuos a la vez que ofrece grandes beneficios a la organización moderna, permitiéndole una mejorara en el presente y ayudar a construir para su futuro, en el que la fuerza de trabajo este organizada para superarse continuamente y esto debe realizarse como un proceso, siempre en relación con el puesto y las metas de la organización.(Siden, 2014)

\section{El Plan de Capacitación del Talento Humano en la Empresa Moderna}

En este sentido, Austee (2013), expone que "Un plan de capacitación para el talento humano en las organizaciones modernas consiste en el análisis de lo que es y lo que representa la formación para el desarrollo en una empresa, cómo se clasifica, como debe administrase e impartirse, esto es, señalando las etapas del proceso para llevarla a cabo y qué actividades se deben realizar en cada etapa del proceso" (p. 112). En este sentido, se hace una codificación de las técnicas que se deben utilizar en la enseñanza, señalando cuál es la más efectiva para el aprendizaje (ob cit). Es decir que son las prácticas y políticas necesarias para reorientar el manejo de los asuntos que tienen que ver con las relaciones humanas del trabajo administrativo; en específico se trata de reclutar, evaluar, capacitar, remunerar y ofrecer un ambiente seguro y equitativo para los empleados de la compañía (Salinero (2015).

Tomando en consideración la definición, se muestra la importancia que tienen las fases para desarrollar un plan de capacitación que facilite el aprendizaje y se pueda aprovechar con mayor efectividad para los trabajadores (Whelpton (1988), de tal manera que las técnicas que se deben utilizar en la enseñanza serán las más eficaces mientras se cumplan las fases de desarrollo del plan de capacitación y de esta manera se puedan poner en práctica las habilidades y conocimientos del personal. A continuación, se describen los pasos para desarrollar un Plan de Capacitación para el talento humano, según Austeen (2013):

Paso 1. Detección de necesidades. consiste en "encontrar las diferencias que existen entre lo que se debería hacer en un puesto y lo que realmente se está haciendo, así como las causas de estas diferencias" (p. 66). En otras palabras, una investigación que se orienta a conocer las carencias que manifiesta un trabajador y que le impiden desempeñar adecuadamente las funciones de su puesto. (Austeen, 2013)

Algunas preguntas que puedes plantear en este momento son las siguientes: ¿Qué conocimientos, habilidades y actitudes requiere el puesto?, ¿Qué se debe lograr?, ¿Qué se debe hacer? ¿Qué conocimientos, habilidades y actitudes requiere la persona que ocupa el puesto? ¿Qué está logrando?, ¿Qué está haciendo? (p. s/n)

Estas preguntas muy generales deben orientarse a investigar los siguientes aspectos, según Austeen, (2013):

Conocimientos y habilidades técnicos que se requieren para el puesto. Habilidades administrativas acerca de cómo organizar el propio trabajo y el de los demás, utilizar los recursos, y lograr los objetivos. Habilidades en relaciones humanas como liderazgo, capacidad de motivar, de comunicación, de trabajar en equipo, etcétera. Habilidades conceptuales como son el análisis y solución de problemas. Habilidad para el logro de resultados. (p. s/n)

Hay que acotar que la detección de necesidades de capacitación puede hacerse a nivel de una persona, de un puesto, de un grupo o de toda la empresa (Austeen, 2013). Es muy importante establecer las necesidades de capacitación en términos de productividad y de una manera objetiva y medible (ob cit). Por ejemplo, se puede establecerlas en aspectos como reducir el margen de error (calidad), incrementar el número de unidades de producto o de prestaciones (cantidad), mejorar la puntualidad (tiempo), reducir los costos de un producto o servicio (costo), (Austeen, 2013)

Paso 2. Clasificación y jerarquización de las necesidades de capacitación. Por su parte, Austeen (2013), señala en este sentido que "una vez detectadas las necesidades de capacitación, el siguiente paso es clasificarlas y jerarquizarlas" (p. 81). Esto permite determinar cuáles son las más urgentes o más importantes. La manera más usual de clasificar las necesidades de capacitación es la siguiente: (ob cit) 
Las que tiene una persona. Las que tiene un grupo. Las que tiene un nivel. Las de un puesto. Las que requieren atención inmediata. Las que requieren solución a futuro. Las que exigen instrucción sobre la marcha. Las que precisan instrucción fuera del trabajo. Las que la empresa puede resolver por sí misma. Las que requieren contratar a capacitadores externos. (p. 81)

Vale destacar que, al clasificar las necesidades de capacitación, se obtienen indicadores sobre quién, cuándo y cómo capacitar. Ya clasificadas se pueden jerarquizar de acuerdo con su importancia o urgencia. (ob cit)

Paso 3. Definición de objetivos. El tercer paso, también según Austeen (2013), "en un proceso de capacitación es establecer los objetivos que se pretende lograr con el programa de capacitación" (p. 89). Este es el momento de fijar que tipo de comportamientos y características se quieren inculcar en los participantes después de que el programa haya sido impartido. Lo que se quiere también es establecer qué conocimientos se les proporcionará o qué actitudes se buscan (ob cit).

En este sentido, es necesario establecer con precisión la conducta deseada y las condiciones en que debe producirse de la manera más clara, alcanzable y medible Siden (2014), comenta al respecto que "esto sirve como norma que permitirá evaluar el rendimiento individual y el programa" (p. 12). Como se dijo anteriormente, se deben establecer los objetivos que se pretende lograr con el programa de capacitación de una manera específica y medible (ob cit).

Hay que recordar que los objetivos sirven como guía para mantenerte en el camino, no se trata únicamente de cubrir un expediente, sino de buscar un resultado concreto que esté lo más relacionado posible con el trabajo (Siden, 2014). Los objetivos deben especificar quién, qué, para cuándo, bajo qué condiciones, cómo medirlos, y a qué costo en tiempo y/o dinero. Un aspecto importante a destacar es que existen diferentes clases de objetivos, como se muestra en la siguiente lista, expuesta por Austeen (2013):

a) Objetivos de productividad: que buscan lograr mejoras medibles en calidad, cantidad, tiempo y costo de operación.b) Objetivos de desempeño: que buscan mejorar el desempeño individual de los participantes. c) Objetivos de habilidades o destrezas: que buscan desarrollar o mejorar las habilidades o destrezas de los participantes. d) Objetivos de conocimientos: que buscan incrementar la familiaridad, los conocimientos o la comprensión de los participantes con respecto a un tema.e) Objetivos del dominio afectivo: que buscan modificar actitudes, valores o emociones de los participantes.(p. 88)

Paso 4. Elaboración del programa. Para elaborar un programa de capacitación tal y como afirma Austeen (2013), se tienen que responder las preguntas: “¿Qué? (Contenido). ¿Cómo? (Técnicas y ayudas). ¿Cuándo? (Fechas y horarios). ¿A quién? (Destinatarios). ¿Quién? (Instructor). ¿Cuánto? (Presupuesto)” (p. 88). Según lo anterior es necesario recordar que es muy importante que exista congruencia entre las necesidades de capacitación, el contenido del programa y los objetivos planteados. (ob cit)

Paso 5. Ejecución. Luego de diseñar y elaborar el programa de capacitación se debe llevar a la práctica la ejecución, es decir, ha llegado el momento en el que el instructor, utilizando cierta metodología y apoyándose en auxiliares didácticos, imparte los contenidos a los destinatarios en el lugar, horario y fechas programadas, a un cierto costo (Austeen, 2013). En este paso es importante cuidar los siguientes aspectos, los cuales se mencionan a continuación:

Organizar en una secuencia lógica y didáctica los contenidos. Organizar las sesiones de aprendizaje y los materiales que se utilizarán. Sensibilizar a los participantes con respecto a los objetivos, contenidos, etcétera. Motivar e involucrar a los participantes. Establecer una buena comunicación con los participantes. Entender y mantener el control del grupo. Vigilar el desarrollo del programa para hacer los ajustes necesarios. (p. 88)

Paso 6. Evaluación. Para saber si los objetivos que se plantearon fueron alcanzados es necesario realizar una evaluación que permitirá medir los resultados del programa de capacitación (Austeen, 2013). Tomando en cuenta lo anterior, la evaluación debe informar sobre cuatro aspectos básicos, según Austeen: La reacción del grupo y de cada participante en cuanto a actitudes. El conocimiento adquirido, es decir, qué aprendió y en qué grado. La conducta, es decir, qué comportamientos se modificaron. Los resultados específicos posteriores al curso. (Austeen, 2013)

La evaluación puede hacerse en tres etapas, tal y como lo describe Austeen (2013):

Al inicio del curso, para diagnosticar y medir el nivel de conocimientos de los participantes antes del curso en relación con los temas que se van a tratar durante el mismo) Durante el curso, para conocer los progresos de los participantes a lo largo del programa y hacer los ajustes necesarios. c) Al final del curso, para medir el grado en que se cumplieron los objetivos. (p. 88) 
Por otra parte, según el autor, también debe evaluarse el curso de capacitación en los siguientes aspectos: actuación del instructor, instalaciones, técnicas y auxiliares didácticos, duración del curso, contenido, servicio de cafetería, el lugar en el que se impartió, entre otros.

Esta retroalimentación servirá para la elaboración de nuevos programas. (Chiavenato, 2013)

Hay que recordar de la misma forma que lo más importante es que los participantes apliquen en su trabajo cotidiano los conocimientos y habilidades adquiridos en el curso. Esa es la verdadera evaluación. Por esto, se puede pensar en el seguimiento como una última etapa del proceso de capacitación. (Austeen, 2013)

\section{Estrategias Organizacionales para fortalecer la capacitación del talento humano en la empresa moderna}

El termino estrategia define la acción que se puede emprender en beneficio de dar diseño, esquema, fase o etapa algún proceso (Salinero, 2015), en tal ámbito de ideas, Carrera y Vásquez (2013), afirman que "permite ser, preciso en la identificación plena de los elementos que se necesitan para perseguir un fin común entre personas, procedimientos o lineamientos que conducen a alcanzar un objetivo en particular" (p. 13).

Dicha acción se convierte en organizacional cuando se trazan líneas horizontales en la fase de planeación de la estrategia, donde se describen responsables, lapsos de tiempos, actividades y los recursos que se van a requerir para cumplir dicha estrategia (Herrera y otros, 2013).

La estrategia organizacional es la creación, implementación y evaluación de las decisiones dentro de una Organización, en base a la cual se alcanzarán los objetivos a largo plazo. (Peña, 2014). Al respecto, Botero (2017), opina que "Consiste en un conjunto total de acciones que se llevan a cabo para lograr un determinado fin" (p. 113).

Ello quiere decir, que es la acción total y que en ella hay inmersa una serie de elementos implícitos y no implícitos que se emprenden en su conjunto para alcanzar un objetivo (ob cit).

En otras palabras, la estrategia organizacional se puede definir tal y como lo expone Castaño (2018), "como el arte y la ciencia de formular, implantar y evaluar decisiones interfuncionales que permitan a la organización llevar a cabo las metas en el corto, mediano y largo plazo" (p. 113). En este sentido, Bolero (2017), expone que:

... se pueden analizar y unificar las definiciones de los planes de capacitación y la estrategia organizacional, ya que ambas están estrechamente relacionadas al ámbito laboral y organizacional de una empresa.

La relación de ambos términos radica en la necesidad de las empresas en diseñar estrategias asociadas a la capacitación de los trabajadores, como una práctica que ayudara a consagrar el alcance de las metas totales que estas suelen proponer ejercicio tras ejercicio económico y que está asociado al mejoramiento de la rentabilidad y operatividad en los mercados donde opera la empresa (p. 273).

Considerando la cita presentada, la filosofía y concepción de la estrategia organizacional en el contexto y dimensión que caracteriza al talento humano, parte de la definición de que los seres humanos poseen capacidades y no recursos de los que se pueden disponer a discreción, son seres con posibilidades de desarrollo e infinito aporte que se ofrece a la organización empresarial para optimizar los procesos y por ende los resultados, los cuales se convierten en mayor y mejor productividad y rentabilidad (Pardo, 2013).

\section{Estrategia Global o Corporativa}

Cuesta (2015), señala que:

La estrategia global o corporativa, recibe también el nombre de estrategia maestra, determina relaciones (comunicación) con el entorno, definiendo actividades en que participa la organización o campo de actividad de ella, estableciendo capacidades distintivas entre los negocios para lograr una sinergia entre ellos, incluyen todo tipo de recursos: físicos, técnicos, financieros, humanos y actividades o competencias de diversas índoles. (p. 26)

Por su parte, Ramírez y Hugueth (2017), asi como El Kadi y Antúnez (2017), manifiestan que "hoy en día, las organizaciones interesadas en mejorar la efectividad de su gestión, han conseguido en la estrategia una herramienta que les permite mantener grupos de trabajo integrados, cumpliendo con los objetivos planificados a través de una comunicación productiva" (p. 365).

En este sentido, se impulsa el crecimiento de la organización con la fuerza de un talento humano informado y en constante comunicación. 


\section{Estrategia de Unidades de Negocio}

Al respecto Cuesta (2015), sostiene:

"De acuerdo a las estrategias de unidades de negocios, las empresas se caracterizan por la multiactividad, con diferentes unidades estratégicas, determina cómo competir en una u otra actividad específica", es decir, es donde verdaderamente combaten las compañías al extender sus capacidades múltiples.

\section{Estrategias Funcionales}

Igualmente Cuesta (2015), al respecto señala:

Por su parte, las estrategias funcionales, se dirigen al logro de una utilización estratégica de los recursos y competencias, de cada área funcional y unidad estratégica de negocio, apoyando a través de la coordinación e integración de políticas en la estrategia corporativa.

Destacando la idea anterior, vale destacar que las concordancias entre éstas forman un patrón importante cuya función en la empresa consiste en establecen las acciones necesarias para emprender con éxito el negocio: recursos humanos, tecnología, producción, marketing y finanzas. (ob cit)

\section{Estrategia Organizacional y Capacitación del Talento Humano}

Los individuos en la empresa desempeñan un rol fundamental en la relación capacitación del talento - estrategia (Cuesta, 2015), pues son estos los que alcanzan la preparación a través del adiestramiento de los diferentes procesos para su posterior ejecución, utilizan y subsanan las tecnologías de que dispone la estructura empresarial y son los encomendados a mantener y modificar con su actuación, el clima organizacional. (Mayfield, Milton; Mayfiled, Jacqueline y Wheeler, Cassandra, 2016).

Por ello es necesario conocer sus actitudes, aptitudes, habilidades y destrezas con el fin de concretar y precisar las diferentes estrategias organizacionales más efectivas, Gutiérrez, Rozo y Flórez, (2019) al respecto, consideran "los modelos de carrera, programas de orientación profesional, planes de sucesión, entre otros" (p. 55); todo lo anterior con el propósito de poder contar con personas que presenten con nuevas habilidades y competencias que lideren procesos de cambio al interior de la organización (Shipside, 2007).

En este marco de ideas; Brazeel (2009), plantea que los profesionales de recursos humanos y los líderes de las organizaciones:

...deberán trabajar juntos con el fin de formar una fuerza de trabajo con las competencias que el nuevo entorno exige: leal, motivada y dispuesta a aprender, lo cual refuerza la generación de ventaja competitiva. Igualmente, los equipos de dirección, junto con los responsables de recursos humanos, deben contar con las competencias (racionales e intuitivas) y las dinámicas de trabajo necesarias para valorar adecuadamente el capital humano de sus empresas, con el fin de garantizar su aporte a la estrategia del negocio (p. 2)

Según lo reseñado en la cita, en un ambiente global, belicoso como el actual, esta labor demanda actividades y labores liderizadas por individuos que en las organizaciones conozcan si su talento humano en la actualidad está dispuesto a favorecer el logro y al sostenimiento de la ventaja competitiva, mediante la información que ofrece el área de recursos humanos, la cual debe ser integrada a los planes estratégicos corporativos (Mayfield, Mayfiled y Wheeler, 2016).

En este sentido, la labor de estos líderes empresariales es alcanzar con la capacitación ofrecida la adquisición de habilidades, destrezas y competencias para aportar a la estrategia organizacional, lo que envuelve mostrarse de acuerdo con la influencia de todas las variables culturales características y presentes en la organización empresarial para el desarrollo de la estrategia y, por ende, en su competitividad (Botero, 2017).

\section{CONCLUSIÓN}

Para la aplicabilidad óptima de las estrategias organizacionales como técnica para fortalecer los planes de capacitación en la empresa moderna resulta imprescindible crear todas las condiciones que permitan mejorar las competencias laborales en los empleados de la organización, en este sentido, está íntimamente relacionado con la manera en que se concibe al sujeto, el cual posee una experiencia y un valioso conocimiento por lo que a la vez que es receptor, es también emisor de conocimiento mediante la información adquirida por medio de la comunicación. (Brazeel 2009). 
Resalta en este sentido, el proceso de aprendizaje activo y dinámico, que considera la capacitación en función de enseñar a reflexionar de forma creadora e innovadora al sujeto a través de técnicas ofrecidas por los diversos enfoques previstos en las estrategias organizacionales (Casta, 2015), como lo son: las estrategias organizacionales globales o corporativas que determinan las relaciones (comunicación) con el entorno, igualmente las estrategias de Unidades de Negocio.

caracterizadas por la multiactividad (Casta, 2015), es decir, la relación con diferentes unidades estratégicas, lo que determina cómo competir en una u otra actividad específica, en este sentido, donde verdaderamente combaten las compañías al extender sus capacidades múltiples y, por último las estrategias funcionales, las que se dirigen al logro de una utilización estratégica de los recursos y competencias, de cada área funcional y unidad estratégica de negocio, apoyando a través de la coordinación e integración de políticas en la estrategia corporativa para lograr una nueva práctica transformadora. (Casta, 2015)

Finalmente, es importante resaltar que las estrategias organizacionales han sido diseñadas sobre la base de metodologías de trabajo que favorezcan la indagación de desarrollo próximo para hacer real el perfeccionamiento potencial del talento humano, donde los procesos de acompañamiento consecuente a la acción del empleado se catequizan en mediador simbólico para alcanzar el estado deseado.

\section{BIBLIOGRAFÍA}

1. Aquino, M. (2016). Dirección Estratégica de Recursos Humanos. Gestión por Competencias. 1er. Ed, Editorial: Granica.

2. Alles, O. (2013). Capacitación del personal. Editorial: Mc Graw Hill. México.

3. Austeen, N. (2013). Los grupos, los equipos y la organización. Editorial Pretince Hall.

4. Barreto, M. (2010). Las funciones de planificación y control. Editorial: Mc Graw Hill. México.

5. Barthes, K. (2009). La capacitación, un recurso dinamizador de las organizaciones. Ediciones E.P.S.O.

6. BrazeeL, Ship. (2009). Human resource's strategy for the future. En: Oil and Gas Journal. 2009. Vol. 107, no. 21.

7. Bolton, J. (1971). Las Simulaciones. Ediciones Prentice Hall.

8. Botero, Luz Dary. (2017). El liderazgo en la gestión administrativa como impulsor de la estrategia para la competitividad internacional empresarial. En: Revista Ciencias Estratégicas. Julio-diciembre, 2017. vol. 25, no. 38, Disponible en: http://www.redalyc.org/articulo. oa?id=151354939001.

9. Carrera, M. y Vázquez, G. (2013). Planteamiento y Análisis de investigaciones, Caracas: El Dorado.

10. Carruzo, E. (2015). Administración en Tiempos de Grandes Oportunidades, Editorial Norma, Bogotá Colombia.

11. Castaño, Luisa. (2018) Relación entre alineación estratégica, cultura organizacional y procesos de gestión de talento humano que contribuyen al desarrollo de Distribuciones Pedimax S.A.S. sede Dosquebradas. Trabajo de grado Administradora de Empresas. Pereira: Universidad Católica de Pereira, 2018. 81 p. Disponible en: http://repositorio.ucp. edu.co/bitstream/10785/5087/1/DDMAE82.pdf

12. Chiavenato, I. (2013). Administración, proceso administrativo. (3를 ed.) México: McGraw - Hill Interamericana de México, S.A.

13. Cuesta, A. (2015). Gestión del talento humano y del conocimiento. (Quinta edición). Ediciones ECOE. Colombia.

14. Dessler, G. (2006). Administración del Personal. 6ta. Edición México. Prentice-Hall Hispanoamericana.

15. Dolan, K., Sehuler, M., y Valle, J., (2011). La Educación en la Empresa, aprendiendo en contextos organizacionales, España, Granica.

16. El Kadi, O; Antúnez, Y. (2017). Consiliencia De La Autotomía Caudal Como Estrategia Gerencial. CICAG, [S.I.], v. $15, \quad$ n. 1 , p. 173 - 191, sep. 2017. ISSN 1856-9439. Disponible en: http://ojs.urbe.edu/index.php/cicag/article/view/323>. Fecha de acceso: 04 enero. 2020.

17. Frody, R. (2016). El futuro de la Dirección de Recursos Humanos, Barcelona, Gestión.

18. Gutiérrez, Carlos; Rozo, Astrid y Florez, Alexander (2019). Direccionamiento estratégico, una estrategia organizacional con alto impacto en el desarrollo laboral. En: Aibi Revista de Investigación, Administración e Ingeniería. 2019. vol. 7, no. 1, p. 8-15. Disponible en: https:// revistas.udes.edu.co/aibi/article/view/544/pdf

19. Herrera, S., Medina, L. y Coronado, P. (2013). Trabajo de Grado titulado: Propuesta de estrategias organizacionales de capacitación para el manejo de maquinarias TOLVAS y empacadoras en la empresa Agropecuaria Gramolca C, A., ubicada en Cagua, Estado Aragua" Trabajo de Grado No Publicado.

20. Hurtado, P. (2012). Planteamiento y Análisis de investigaciones, Caracas: El Dorado. 
21. Mayfield, Milton; Mayfiled, Jacqueline y Wheeler, Cassandra (2016). Talent development for top leaders: three HR initiatives for competitive advantage. In: Human Resource Management International Digest. 2016. vol. 24, no. 6, p. 4-7. DOI 10.1108/HRMID-07-2015-0120.

22. Mondy, R. (2005). Administración de Recursos Humanos. Pearson/Prentice Hall. México.

23. Pardo, L. (2013). Gestión del talento humano. Revista arbitrada del centro de investigación y estudios gerenciales A.C. Volumen 4, №1. Barquisimeto - Venezuela. (Pp. 60-71).

24. Peña, L. (2014). Estudio titulado: Diseño de un Manual de Capacitación de Personal para el Departamento de Recursos Humanos en la Empresa Ávila Química, S.A. en Valencia, Estado Carabobo, Trabajo de Grado No Publicado.

25. Ramírez, R. y Hugueth, A. (2017). Modelo de comunicación productiva para las organizaciones de salud pública en Venezuela. Revista Opción. Volumen 33, №83. Maracaibo - Estado Zulia, República Bolivariana de Venezuela Barquisimeto - Venezuela. (Pp. 305-335).

26. Salinero, M. (2015). Estudio titulado: Diseño de un Plan de Formación como estrategia de desarrollo empresarial: estructura, instrumentos y técnicas, Trabajo de Grado no Publicado.

27. Schuler, R.S. (1992). Strategic Human Resource Management: Linking people with the needs of the business. Organizational Dynamics, 21 (1) 18-32.

28. Shipside, Steve. (2007) How to keep the cream of the crop? En: Newspaper Technique. Julio, 2007. p. 14-17.

29. Siden, M. (2014). Como evaluar las acciones de capacitación, Ediciones Granica, s.a., Argentina.

30. Whelpton, G. (1988). La organización requedida. Ediciones Granica.

31. Menéndez, M. (2016) Importancia de la Comunicación Relaciones Públicas y Tic'S en las Organizaciones del Siglo XXI http://ojs.formacion.edu.ec/index.php/rif/article/view/42 DOI: https://doi.org/10.34070/rif.v4i1 